\title{
Hospital Acquired Infections at the Service of Pediatric Surgery in Gabriel Touré Academic Hospital, Bamako, Mali
}

\author{
Y. Coulibaly1, 0. Kone², I. Amadou1, T. H. M. Diop³, 0. Coulibaly'1, A. Doumbia1, B. Kamaté1, \\ H. Ouologuem', D. Konaté4, M. Coulibaly ${ }^{1}$, B. Maiga ${ }^{4}$, M. I. Mangané ${ }^{3}$ \\ ${ }^{1}$ Pediatric Surgery, CHU Gabriel Touré, Bamako, Mali \\ ${ }^{2}$ Community Health Divisions, INRSP, Bamako, Mali \\ ${ }^{3}$ Anesthesia Intensive Care, University Hospital Gabriel Touré, Bamako, Mali \\ ${ }^{4}$ Department of Pediatrics, CHU Gabriel Touré, Bamako, Mali \\ Email: *coulibalyyacak@yahoo.fr
}

How to cite this paper: Coulibaly, Y., Kone, O., Amadou, I., Diop, T.H.M., Coulibaly, O., Doumbia, A., Kamaté, B., Ouologuem, H., Konaté, D., Coulibaly, M., Maiga, B. and Mangané, M.I. (2020) Hospital Acquired Infections at the Service of Pediatric Surgery in Gabriel Touré Academic Hospital, Bamako, Mali. Open Journal of Pediatrics, 10, 185-193. https://doi.org/10.4236/ojped.2020.101018

Received: December 31, 2019

Accepted: March 6, 2020

Published: March 9, 2020

Copyright ( 2020 by author(s) and Scientific Research Publishing Inc. This work is licensed under the Creative Commons Attribution International License (CC BY 4.0).

http://creativecommons.org/licenses/by/4.0/ (c) (i) Open Access

\begin{abstract}
Introduction: Health care-associated infection is an infection acquired during the care delivered in the hospital or in any other care establishment which was neither present, nor in incubation at the admission of the patient or at the time of delivering the care or which does not manifest itself only after the patient has been discharged or an infection contracted by the professional in the course of his duties. Objectives: To identify mean causative germs and to determine their sensitivity to antibiotics and to identify the risk factors of health care-associated infection. Material and methods: It was about a prospective longitudinal study conducted from November 1, 2017 to April 1, 2018 (6 months) in all children admitted to the pediatric surgery service. Non-consenting parents and outpatient necrosectomy case have not been included in this study. Results: This study included 200 patients, of whom 30 experienced the hospital acquired infections with an infection rate of $15 \%$. The surgical site infection was the most common type, with 16 cases (53.3\%), followed by burns in 13 cases $(43.3 \%)$ and urinary tract infection in 1 case (3.3\%). The average age of patients with infection was $56.33 \pm 48.66$ months with extremes of 1 and 180 months. The sex ratio was 1.30 for infected patients and was 1.83 for uninfected patients. The main bacteria responsible for surgical site infection were: Escherichia coli (4 cases), Acinetobacter baumanii (3 cases), Klebsiella pneumoniae (2 cases), Staphylococcus aureus (2 cases), Enterobacter cloacae (1 case), Pseudomonas aeruginosa (1 case) and Enterobacter faecalis ( 1 case). Among burn patients, the most frequent germs were: Acinetobacter baumanii (7 cases), Klebsiella pneumoniae (6 cases), Staphylococcus aureus (6 cases), Escherichia coli (4 cases), Pseudomonas ae-
\end{abstract}


ruginosa (2 cases) and Enterobacter faecalis (2 cases). Escherichia coli was noted only in the case of urinary tract infection. Antibiotics tested were: amoxicillin, amoxicillin associated with clavulanic acid, ceftriaxone, imipenem, gentamicin and ciprofloxacin. Germs were resistant to amoxicillin in $88.9 \%$ of cases, sensitive to the amoxicillin-clavulanic acid combination in $85 \%$ of cases, sensitive to tobramycin and gentamicin. Recruitment methods and length of hospitalization were the risk factors noted. Conclusion: Massive awareness among all healthcare providers should be conducted on the adoption of standard precautions for the prevention of health care-associated infections and on the rules of antibiotics prescription to reduce the incidence of health care-associated infection and slow the resistance of germs to antibiotics.

\section{Keywords}

Infection, Germs, Sensitivities, Risk Factors, Child, Mali

\section{Introduction}

The health care-associated infection (HAI) is a reflection of the care service quality in a health system. Its frequency, its consequences and its cost are high enough and serious for it to be considered as a public health problem, in order to be prevented. Its prevalence in Southeast Asia is 9\% [1]. It is about of $15.5 \%$ in developing countries [2]. Besides prolonging hospital stays, healthcare associated infections are also responsible for long-term disabilities, high additional financial burden, increased mortality, high costs for health systems and an emotional impact for patients and their families. The frequency of occurrence of HAI varies with patient care procedures, invasive devices and the type of service, intensive care being the most risky sector. The risk of a HAI depends on factors relating to the pathogen, host and environment. The infection is most often microbial. In developing countries, the magnitude of the problem remains underestimated or even unknown largely because the diagnosis of HAI is complex and surveillance activities to guide interventions to require expertise and resources [3]. The lack of study in the healthcare associated infections in children has motivated the realization of this study. Our objectives were to determine the main responsible of infection germs associated with care and sensitivity to antibiotics and to identify the risk factors of care-associated infection.

\section{Material and Methods}

This was a longitudinal prospective study conducted from November 1, 2017 to April 1, 2018 in all children admitted to the pediatric surgery service. Non-consenting parents and necrosectomy cases have not been included in this study. The nosocomial infection was identified according to the criteria defined by the Center of Disease Control of Atlanta. A period of 48 hours was held between admission and the beginning of the infection. A sample was taken in all 
suspected cases of infection. These samples were sent directly to the medical biology laboratory. A questionnaire was established for data collection. The main studied parameters were age, gender, recruitment mode, ASA class, the presence of anemia, nutritional status, the presence of a venous catheter, the urinary catheter, and the duration and the hospitalization category.

Data were entered and analyzed on SPSS (version 10.0) and the Epi-Info software (version 7.0). The results were discussed with the chi $^{2}$ and Fisher statistical tests for qualitative variables and Kruskal Wallis ANOVA for quantitative variables. The level of significance was $\mathrm{p}<5 \%$.

\section{Results}

Our study included 200 patients, of whom 30 experienced a hospital acquired infections. The infection rate was of $15 \%$. The surgical site infection was the most common type (16 cases: 53.3\%), followed by the burn infection in 13 cases (43.3\%) and urinary tract infection in 1 case (3.3\%). The average age of patients with infection was $56.33 \pm 48.66$ months with extremes of 1 and 180 months and the uninfected patients was $64.94 \pm 56.88$ months with extremes of 1 and 180 months. Among patients with an acquired infection, boys outnumbered 17 (56.7\%) with only 13 girls $(43.3 \%)$. The sex ratio was 1.30 for infected patients and was 1.83 for uninfected patients.

A germ was found in pus cytobacteriological examination in 29 patients (96.7\%) against only 1 case (3.3\%) at cytobacteriological examination of urine. All cultures were positive. Isolated germs are listed in Table 1.

The main bacteria responsible for surgical site infection were: Escherichia coli (4 cases), Acinetobacter baumanii (3 cases), Klebsiella pneumoniae (2 cases), Staphylococcus aureus (2 cases), Enterobacter cloacae (1 case), Pseudomonas aeruginosa (1 case) and Enterobacter faecalis (1 case).

In burn patients the prominent germs were: Acinetobacter baumanii ( 7 cases), Klebsiella pneumoniae (6 cases), Staphylococcus aureus (6 cases), Escherichia coli (4 cases), Pseudomonas aeruginosa (2 cases) and Enterobacter faecalis (2 cases). Escherichia coliwere noted only in the case of urinary tract infection.

Antibiotics tested were: amoxicillin, amoxicillin-clavulanic acid, ceftriaxone, imipenem, gentamicin and ciprofloxacin.

Thirteen (13) strains were tested at amoxicillin; a single strain of Escherichia coli was resistant in $92 \%$ of cases.

As for amoxicillin-clavulanic acid, of 22 germs tested, eight were sensitive (4 strains of Escherichia coli and 4 strains of Staphylococcus aureus).

Ceftriaxone was active on 11 of the 16 strains tested (69\% of sensitivity). All strains of Acinetobacter baumanii (10 strains) and three strains of Klebsiella pneumoniae were resistant to imipenem. But it was active against the other tested bacteria (17 strains).

The total strain sensitivity to gentamicin was 58\% (21/36). All Acinetobacter baumanii strains tested were resistant (7/7). 
Table 1. Isolated bacteria.

\begin{tabular}{ccccc}
\hline Isolated germs & Alone & associate & NOT & $\%$ \\
\hline Acinetobacter baumanii & 8 & 2 & 10 & $23.81 \%$ \\
Pseudomonas aeruginosa & 2 & 1 & 3 & $7.14 \%$ \\
Klebsiella pneumoniae & 5 & 3 & 8 & $19.05 \%$ \\
Escherichia coli & 5 & 4 & 9 & $21.43 \%$ \\
Staphylococcus aureus & 8 & 0 & 8 & $19.05 \%$ \\
Enterobacter faecalis & 1 & 2 & 3 & $7.14 \%$ \\
Enterobacter cloacae & 1 & 0 & 1 & $2.38 \%$ \\
Total & 30 & 12 & 42 & $100 \%$ \\
\hline
\end{tabular}

Sensitivity for ciprofloxacin was $42 \%$ (16/38), but we noted a significant resistance of tested strains: Klebsiella pneumoniae (100\%), Pseudomonas aeruginosa (100\%), Acinetobacter baumanii (90\%), and Escherichia coli (55\%) The main risk factors are listed in Table 2. Infection was more common in infants. Out of a total of 79 infants, 13 developed an infection. Boys were more frequent in the group of infected patients. According to the method of recruitment, 26 out of 63 emergency patients presented an infection. For the duration of hospitalization out of 30 patients hospitalized for more than 15 days, 11 presented an infection.

After the treatment we noted 9 deaths (4.5\%).

\section{Discussion and Comments}

During this work we have been facing to some difficulties among which the inability to perform cytobacteriological examination of samples during holidays and difficulties carrying samples to private laboratories during operating hours.

We collected 200 patients' files while 90 was the necessary and sufficient sample size by considering the rate of nosocomial infection of $12.2 \%$ and the risk of error of $5 \%$. Our $15 \%$ infection rate is higher than the $4.3 \%$ of Smetana [4] in the Czech Republic and 9.6\% of Antonioli [5] in Italy. The infection is multifactorial; it would be difficult to explain this difference, but some factors may influence the occurrence of infection in our context. They were the precarious hygiene conditions, pre, intra and postoperative asepsis, the socio-economic deficient conditions and the poor preparation of patients undergoing emergency.

The most frequent found germ in our series was Acinetobacter baumanii (23.8\%) in contrast to the series of Afle in Benin [6]. The seeds of our series reflect the microbial flora of the service where the digestive surgery is dominant. These results differ from those reported in 2015 in the National Hospital and University Center Koutoukou Hubert Maga Cotonou (Benin) where Klebsiella pneumoniae, Escherichia coli, Acinetobacter spp, Proteus mirabilis and Enterobacter cloacae represented 53\% [7]. Merzouguia et al. [8] in their study isolatedin order of frequency the following germs: $K$. Pneumonia (41.0\%), followed by E. coli $(24.3 \%)$ and $S$. aureus $(14.1 \%)$. Concerning the sensitivity of bacteria to 
Table 2. Risk factors.

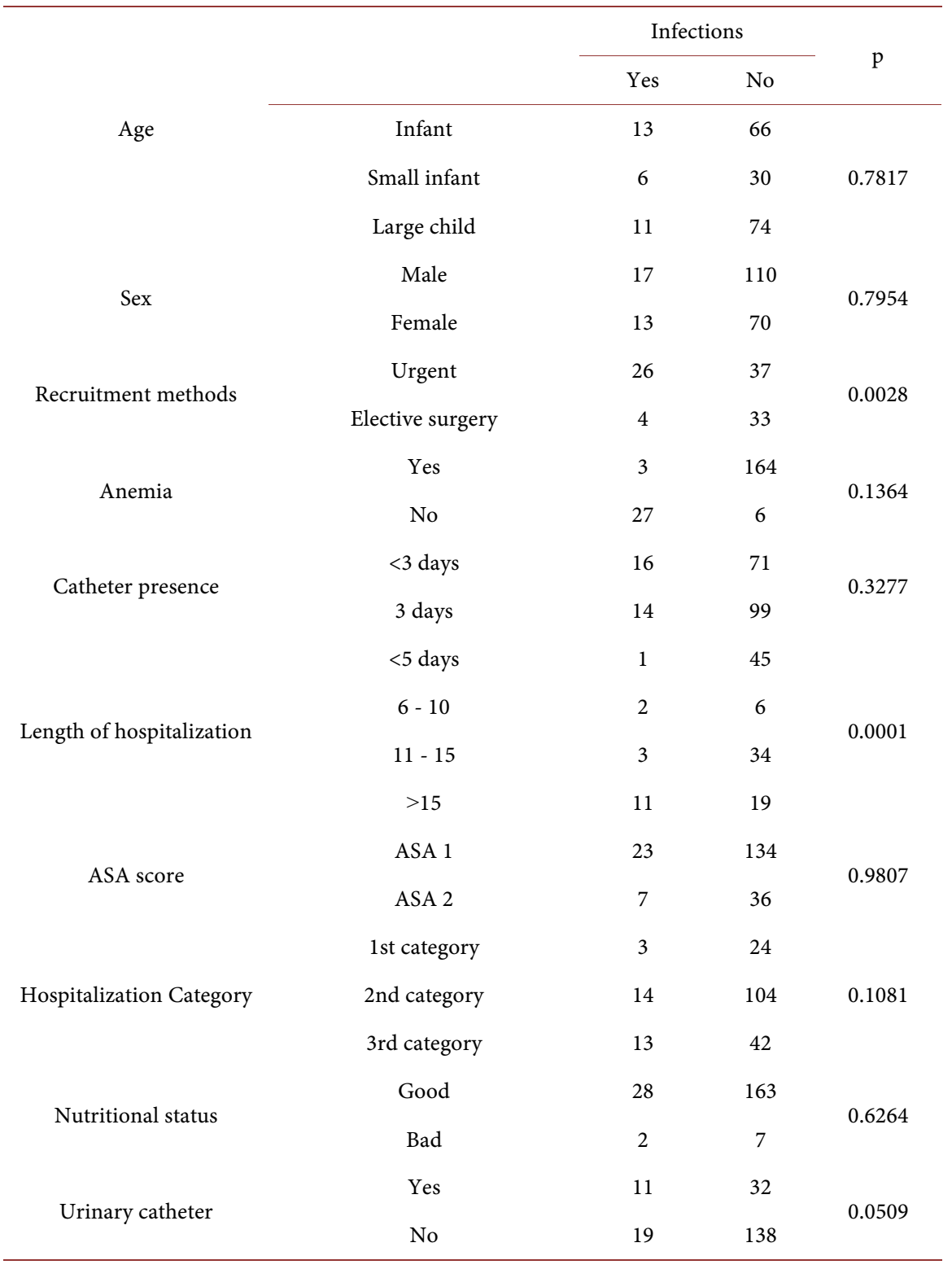

antibiotics tested Acinetobacter baumanii, Klebsiella pneumoniae, Pseudomonas aeruginosa were resistant to ceftriaxone in $100 \%$ of cases. A strain of Escherichia coli was sensitive to $9.52 \%$.

All seeds were resistant to amoxicillin except a strain of Escherichia coli sensitive to $11.1 \%$. The overall resistance of bacteria to amoxicillin was $88.9 \%$. That of Escherichia coli to this antibiotic has been reported by some authors as Bercion [9]. This resistance could be explained by the absence of antibiotic prescribing strategies, self-medication, ignorance of antibiotic resistance problems in most of the personnel of dispensaries that are not generally pharmacists and illegal sale pharmaceuticals.

Dougnon et al. [10] found that the quality of discs of antibiotics used for carrying out antibiogram could be a factor promoting bacterial resistance. There- 
fore, it is important to end the self-medication and the use of poor quality antibiotics from an awareness and continuous information of all health actors.

The germs were sensitive to the amoxicillin-clavulanic acid combination in 85\% of cases except Acinetobacter baumanii which was resistant in $100 \%$.

All seeds were sensitive to tobramycin and gentamicin except Acinetobacter baumanii that was resistant to gentamicin in $100 \%$ of cases. The imipenem and ertapenem were active on all germs. Ciprofloxacin was active in $38.10 \%$ of germs but a resistance against different strains of Acinetobacter baumanii, Escherichia coli and Klebsiella pneumoniae was observed in respectively $40.91 \%, 22.73 \%$ and $31.82 \%$ of cases. We are seeing a decrease in the sensitivity of bacteria to this antibiotic. This could be explained by the inappropriate and abusive use of this molecule in the treatment of certain infections supposed typhoid or urinary often. Increasing the percentage of Escherichia coli and Klebsiella pneumoniae resistant to third generation cephalosporins in bacteremia result, presumably, of the misuse of these antibiotics [11].

All seeds were resistant to tetracycline in $100 \%$ of cases. The negative GRAM bacilli were resistant to chloramphenicol in $80 \%$ of cases except Escherichia coli and Klebsiella pneumonia in respectively $20 \%$ and $30 \%$ of cases. The GRAM positive cocci have been sensitive in $100 \%$ of cases.

The sulfamethoxazole + trimethoprim were active on Escherichia coli in $7.14 \%$ of cases. Other negative bacilli GRAM were resistant in $100 \%$ of cases. Staphylococcus aureus was sensitive $33 \%$ of cases. This germ was sensitive to colistinin $100 \%$ and Escherichia coli were resistant in $12.5 \%$ of cases.

Antimicrobial resistance occurs naturally in time, usually as a result of genetic changes but the improper or excessive use of antibiotics accelerates the process [12]. According to the Institute of Health Surveillance and Alert Network, Investigation and Surveillance of Nosocomial Infections, the spread of BMR results from interactions between four factors: 1) the selection pressure exerted by the excessive use of antibiotics; 2) the transmission of resistant strains of patients through patient care and caregivers; 3 ) epidemic resistant strains, as applicable, may have an advantage in terms of transmission; 4) the susceptibility to infection in some patients [13].

Regarding risk factors, sex and age were not decisive in the occurrence of infection in our series. Some authors believe that surgical site infection is more common in girls than in boys because of the importance of subcutaneous fat.

The ASA score is predictive of nosocomial infection. It was not an infection risk factor in our series as in that of Benchouk in Algeria [14].

Regarding the method of recruitment, the rate of nosocomial infection of patients urgently admitted was $13 \%$ against $2 \%$ of those admitted in schedule program with $\mathrm{p}<0.5$. The emergency context favors the CAI occurrence due to the lack of necessary preparation and of the fragility of the patient. Some authors believe that there is no link between the method of recruitment and CAI occurrence [15].

Anemia is recognized as a factor weakening the patient due to tissue hy- 
po-oxygenation therefore promotes infection. It was not decisive in the occurrence of HAI in our series ( $\mathrm{p}>0.05)$.

Poor nutritional status is accompanied by a reduction of the body's defenses because of serum hypo protein and hypo albumin. Poor nutritional status was not a risk factor in our study probably due to the small number of undernourished patients.

The installation of a venous catheter is an invasive procedure that may cause a HAI especially if that period is extended. The presence of a thrombus at the catheter is associated with a significant increase in the rate of colonization and catheter-related infections. The presence of a catheter was not decisive in our series $(\mathrm{p}>0.05)$.

The urinary catheter when it is put in aseptic conditions can promote infection. The urinary catheter is the main risk factor for nosocomial UTIs [15]. In our series it did not influence the occurrence of infection in contrast to the study of Chemsi [16].

The category of hospitalization did not influence the occurrence of HAI. In our opinion the quality of care should not vary according to category of hospitalization.

The average hospital stay was $13.84 \pm 14.02$ days for infected patients and 5.27 \pm 2.24 days for non-infected patients. It was 3 times lengthened in case of infection. It was a risk factor of CAI as in the series of Merzouguia and Kakupa 1 [8] [17]. The healing of the wound delay may explain the prolongation of hospital stay.

\section{Conclusion}

Massive awareness among all healthcare providers should be conducted on the adoption of standard precautions for the prevention of HAI and on the rules of prescription and use of antibiotics to reduce the incidence of HAI and slow down the resistance of germs to antibiotics.

\section{Conflicts of Interest}

The authors declare no conflicts of interest regarding the publication of this paper.

\section{References}

[1] Ling, M.L., Apisarnthanarak, A. and Madriaga, G. (2015) The Burden of Healthcare-Associated Infections in Southeast Asia: A Systematic Literature Review and Meta-Analysis. Clinical Infectious Diseases, 60, 1690-1699. https://doi.org/10.1093/cid/civ095

[2] Allegranzi, B., Nejad, S.B., Combescure, C., Graafmans, W., Attar, H. and Donaldson, L. (2011) Burden of Endemic Health-Care-Associated Infection in Developing Countries: Systematic Review and Meta-Analysis. The Lancet, 377, 228-241. https://doi.org/10.1016/S0140-6736(10)61458-4

[3] Allegranzi, B. and Pittet, D. (2008) Preventing Infections Acquired during 
Health-Care Delivery. The Lancet, 372, 1719-1720. https://doi.org/10.1016/S0140-6736(08)61715-8

[4] Smetana, J., Čečetková, B. and Chlíbek, R. (2014) Prevalence Study of Nosocomial Infections in University Hospitals in the Czech Republic. Epidemiologie, Mikrobiologie, Imunologie, 63, 251-258.

[5] Antonioli, P., Manzalini, M.C., Stefanati, A., Bonato, B., Verzola, A., Formaglio, A. and Gabutti, G. (2016) Temporal Trends of Healthcare Associated Infections and Antimicrobial Use in 2011-2013, Observed with Annual Point Prevalence Surveys in Ferrara University Hospital, Italy. Journal of Preventive Medicine and Hygiene, 57, 135-141.

[6] Cyr Doscoph Afle, F., Quenum, K. and Roch Johnson, S.H. (2018) État Christian Places of Healthcare Associated Infections in Two Public Hospitals in Southern Benin (West Africa): University Hospital Area of Abomey/Sô-Ava and Hospital Cotonou Zone 5. Journal of Applied Biosciences, 121, 12192-12201.

https://doi.org/10.4314/jab.v121i1.9

[7] Ouendo, E.M., Saizonou, J., Degbey, C., Glélé Kakai, C., Glélé, Y. and Makoutode, M. (2015) Infectious Risk Management Associated with the Care and Services to the National Hospital and University Center koutoukou Hubert Maga Cotonou (Benin). The International Journal of Biological and Chemical Sciences, 9, 292-300. https://doi.org/10.4314/ijbcs.v9i1.26

[8] Merzouguia, L., Ben Helel, K., Hanachia, H., Metjaouel, H., Ha Barkallah, B. and Rejeb, B. and Said-Latiri, H. (2018) Risk Factors for Nosocomial Bacterial Infection in a Neonatal Center of the Tunisian Center. "Case-Control Study": About 184 Cases. Journal of Paediatrics and Childcare, 31, 18-26. https://doi.org/10.1016/j.jpp.2017.12.001

[9] Bercion, R., Gaudeuille, A., Mapouka, P.A., Behounde, T. and Guetahoun, Y. (2007) Surgical Site Infections in Orthopedic Surgery Department of the Bangui Community Hospital, Central African Republic. Bulletin de la Société de Pathologie Exotique, 100, 197-200.

[10] Dougnon, T.V., Johnson, R.C., Bankole, H.S., Koudjalé, B., Hounmanou, G., Baba-Moussa, L. and Boko, M. (2016) Performance Evaluation of Three Antibiotic Discs Brands Sold in Benin. Health Sciences and Diseases, 17, 71-78.

[11] Surveillance of Multidrug-Resistant Bacteria in Health Facilities. BMR-Raisin Network, France. 2015 Results.

[12] World Health Organization (WHO) (2014) Antimicrobial resistance: Global Report on Surveillance 2014. http://apps.who.Int/iris/bitstream/10665/112642/1/9789241564748 eng.pdf?u=1

[13] Desenclos, J.-C. and Brun-Buisson, C. (2011) The Fight against Healthcare Associated Infections: Progress Certainly, But We Must Continue the Mobilization! $117 \mathrm{~ns}$.

[14] Benchoukt, G.S. (2013) Prevalence of Nasal Carriage of Staphylococcus Aureus: Its Role in Surgical Site Infection. Constatine.

[15] Wagenlehner, F.M. and Naber, K.G. (2000) Hospital-Acquired Urinary Tract Infections. Journal of Hospital Infection, 46, 171-81.

https://doi.org/10.1016/S0195-6701(00)90821-1

[16] Chemsi, M., Shahid, I., Lehlimi, M., Aalloula, O., Zeroualic, K., Habzi, A. and Benomar, S. (2013) Incidence of Nosocomial Bacterial Infections. Children's Hospital Abderrahim Harouchi CHU Ibn Rushd, Casablanca, Morocco. Journal of Paedia- 
trics and Childcare, 26, 11-18. https://doi.org/10.1016/j.jpp.2012.11.001

[17] Kakupa, D.K., Baudouin Byl, P.K.M. and Wilmet, M.D. (2016) Study of the Prevalence of Nosocomial Infections and Associated Factors in the Two University Hospitals in Lubumbashi, Democratic Republic of Congo: The Case of University Clinics Lubumbashi Hospital Janson Sendwe. Pan African Medical Journal, 24, 275. https://doi.org/10.11604/pamj.2016.24.275.7626 